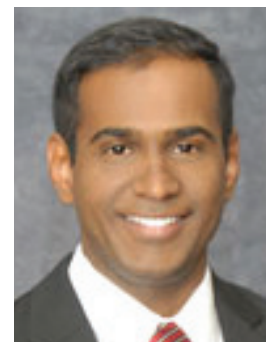

\title{
REMOTE MONITORING OF HEART FAILURE PATIENTS
}

\author{
Arvind Bhimaraj, M.D., M.P.H. \\ Methodist DeBakey Heart \& Vascular Center, The Methodist Hospital, Houston, Texas \\ Weill Cornell Medical School, New York, New York
}

A. Bhimaraj, M.D., M.P.H.

\begin{abstract}
"The Teledactyl (Tele, far; Dactyl, finger - from the Greek) is a future instrument by which it will be possible for us to 'feel at a distance.' This idea is not at all impossible, for the instrument can be built today with means available right now. It is simply the well known telautograph, translated into radio terms, with additional refinements. The doctor of the future, by means of this instrument, will be able to feel his patient, as it were, at a distance...The doctor manipulates his controls, which are then manipulated at the patient's room in exactly the same manner. The doctor sees what is going on in the patient's room by means of a television screen."

-Hugo Gernsback, Science and Invention Magazine, February 1925
\end{abstract}

\section{Abstract}

Heart failure continues to be a major burden on our health care system. As the number of patients with heart failure increases, the cost of hospitalization alone is contributing significantly to the overall cost of this disease. Readmission rate and hospital length of stay are emerging as quality markers of heart failure care along with reimbursement policies that force hospitals to optimize these outcomes. Apart from maintaining quality assurance, the disease process of heart failure per-se requires demanding and close attention to vitals, diet, and medication compliance to prevent acute decompensation episodes. Remote patient monitoring is morphing into a key disease management strategy to optimize care for heart failure. Innovative implantable technologies to monitor intracardiac hemodynamics also are evolving, which potentially could offer better and substantial parameters to monitor.

\section{Introduction}

It has never been more important to evaluate practices that can minimize the economic burden on the U.S. health care system. Heart failure (HF) alone contributes to $\$ 34$ billion dollars in health care expenditure, of which $75 \%$ comes from inpatient care. Acute decompensated HF is the most common reason for admission, and the majority of episodes are in prevalent cases. Clearly, there is a need to predict such episodes and intervene early enough to avoid hospitalization. Economics also have driven regulations in targeting quality parameters of shorter length of stay and lower readmission rate. Moreover, acute decompensation has been strongly associated with future mortality, and strategies to abort such occurrences could potentially translate into improved survival.

\section{Monitoring Beyond the Healthcare Setting}

A snapshot from a clinic visit is rarely reflective of a patient's physiological state at home, hence the need for patient home monitoring. A failing myocardium needs constant and consistent maintenance of a milieu that promotes healing and minimizes negative remodeling. In order to maintain good outcomes, it is incumbent on HF patients to be extremely complaint, vigilant, and in tune with the goals of therapies and care. However, a significant proportion of patients are faced with an acute decompensation of chronic $\mathrm{HF}^{1}$ due to dietary and medication noncompliance leading to congestive symptoms and possible hospitalization. Once admitted, $25 \%$ of patients are readmitted in 1 month, and up to $50 \%$ are readmitted in 6 months. Home monitoring allows clinicians to identify early warning signs of fluid retention and intervene as needed, either by reinstating adherence to optimal diet and medications or increasing diuretic dosing to avoid worsening of symptoms. Various mechanisms of monitoring patients at home and variable combinations and permutations of interventions have been studied. The following is an evidencebased review of the various home monitoring systems for heart failure patients.

\section{Structured Telephone Monitoring}

The basic concept of care extending beyond the health care setting is captured by a simple phone call monitoring strategy wherein patient compliance, symptoms, vital signs, and weight are followed remotely. The DIAL study ${ }^{2-4}$ was one of the first to put to trial Structured Telephone Support (STS), with 1,518 HF patients randomized into either a control group or an intervention group that received STS. In the intervention group, dedicated nurses called every 14 days and adjusted the frequency accordingly thereafter for a year. Predetermined standardized questions were used to assess dyspnea/fatigue, daily weight monitoring, edema progression, dietary and drug therapy compliance, and physical activity. The nurses were only allowed to change the diuretic dose and recommend a nonscheduled medical visit. Nurses used a computer-aided software system to keep a log of conversations and get reminders for phone calls. All study subjects were followed on a 3-month basis irrespective of the unscheduled visits and phone calls. Most (80\%) of these patients had systolic dysfunction and had NYHA class II-III symptoms. Overall, the intervention group had fewer rehospitalizations both in the short term and at $1-3$ years after stopping the intervention.

In a recent meta-analysis, Inglis et al. ${ }^{5}$ reviewed 16 studies utilizing STS and found that there was a nonsignificant trend 

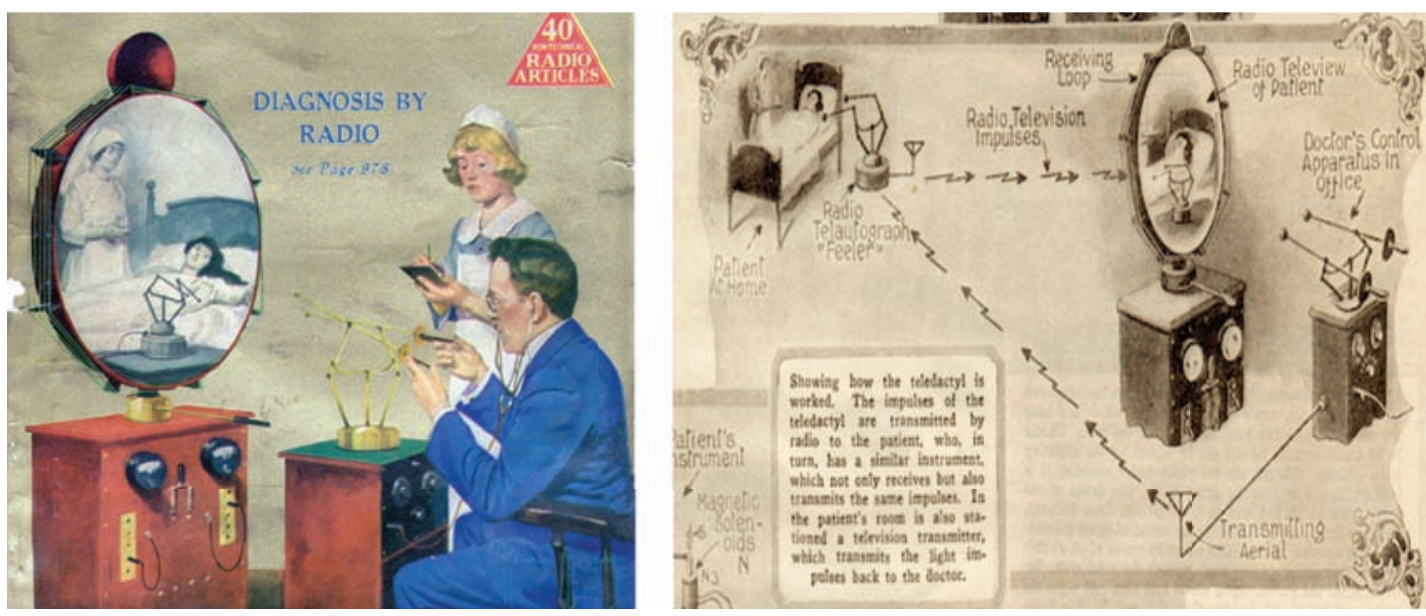

Figure 1. The Teledactyl was an historical concept for telemedicine. From Gernsback H. Science and Invention. 1925 Feb.

towards improved mortality (RR 0.88 [95\% CI 0.76-1.01], $P=0.08$ ) and a significant $23 \%$ reduction in CHF hospitalizations (RR 0.77 [95\% CI 0.68-0.87]). All-cause hospitalizations also were reduced. ${ }^{5}$ Though there was heterogeneity in the various studies, all STS studies included in this meta-analysis had some form of patient education and monitoring. The specifics of intervention in the context of medication changes were not uniform, and some studies had the intervention led by a pharmacist. Of the 16 studies, 6 had reported improved quality of life (with STS) in both overall and physical scores on the Minnesota Living with Heart Failure Questionnaire and the Kansas City Cardiomyopathy Questionnaire.

The impact of STS appears to be related to increased patient contact time that reinforces the importance of compliance with medication and diet regimens. Interestingly, counterintuitive to logic, the intensity of home monitoring utilizing STS did not seem to make a difference in the outcome. In the $\mathrm{COACH}$ trial, ${ }^{6}$ moderate and intensive nursing support after hospital discharge made no significant difference in death and all-cause hospitalization, while in the TEN-HMS study, ${ }^{7}$ STS was as effective as a more intensive and sophisticated monitoring intervention. Of note, both interventions did better than standard of care in this study.

Based on these outcomes and the DIAL study, ${ }^{2}$ which showed that a telephone intervention for 16 months had a sustained positive impact on patients for up to 3 years, STS intervention has the potential to positively affect outcomes during the vulnerable period after discharge.

\section{Monitoring With Home-Based Portable Technology}

The idea of transmitting medical information stems as far back as 1906, when Willem Leister Einthoven sent an EKG over telephone lines. In fact, illustrations of telemedicine can be found as early as 1924, when Hugo Gernsback speculated about telemedicine in his journal, Science and Medicine (Figure 1). Though the futuristic vision of the early 20th century is gradually coming to fruition for HF management, such technological advancements still need to stand the test of evidence-based and cost effectiveness research. Various mechanisms of data transmission utilizing telephonic or Bluetooth transmission have been used.

The Telemonitoring to Improve Heart Failure Outcomes (Tele-HF) study randomized 1,653 subjects within 30 days of an HF hospitalization to a telephone-based interactive voice response system or usual care. The voice response system (Tel-Assurance ${ }^{\circledR}$, Pharos Innovations, Northfield, IL) included a series of questions related to general health and HF symptoms, with patients entering their responses using the telephone keypad. Clinical information such as blood pressure and daily weight was not collected. At the end of 26 weeks, adherence to this intervention was only $55 \%$, and there was no significant impact on mortality or rehospitalizations compared to usual care. Though the authors of this study claimed to have found evidence in a more rigorous study design and found results contradicting a Cochrane review on telemonitoring, ${ }^{8}$ it is important to note that the monitoring in the Tele-HF study had very basic patient information along with dismal compliance. Also, the ability of weight and symptoms alone to predict a decompensation has been shown to be low.

The Trans-European Network-Home-Care Management System (TEN-HMS) study attempted to find a complex algorithm utilizing daily weights to predict worsening HF. ${ }^{9}$ A simple rule-of-thumb algorithm and a moving average convergence divergence (MACD) algorithm were compared. Though the complex MACD algorithm was found to be more specific but less sensitive than a rule of thumb, many episodes of acute decompensations did not appear to be associated with weight gain. On the contrary, a smaller study by Goldberg et al. ${ }^{10}$ reported a $10.4 \%$ absolute and $56.2 \%$ relative reduction in mortality in a monitoring system using symptoms and weight. In spite of these inconsistencies, due to a lack of reliable parameters, management guidelines still recommend daily weight measurements with an alert to an increase of $>2 \mathrm{lbs}$ in a day. ${ }^{11}$ Another large telemonitoring study was the Telemedical Interventional Monitoring in Heart Failure (TIM-HF) trial that used Bluetooth technology to transmit weight, blood pressure, heart rhythm, and a self-assessed health status over a mobile telephone connection. Apart from a structured monthly phone call, there was physician-led medical support available 24 hours a day, 7 days a week. Intervention was provided based on set standards on an ongoing basis. A total of 710 patients were randomized to the monitoring system and usual care. Compliance in the intervention arm was high $-81 \%$ had at least $70 \%$ of daily data transmission. Follow-up at 26 months (on average) showed no difference in overall mortality, cardiovascular mortality, or hospitalizations.

In addition to weight, the TEN-HMS study also monitored blood pressure, heart rate, and rhythm (with a single-lead electrocardiogram) twice daily and transmitted into a hub connected to a conventional telephone line, which then transmitted information through a central web server to each investigator site. The study was halted early due to a significantly higher mortality in the usual care group (reflecting a high-risk population) when compared to either nurse telephone support or the above-described 
telemonitoring system. There was no significant difference in outcomes between the latter two groups. The patient contact time was significantly lower in the usual-care arm, and evidence-based medications were more frequently used in both the nursebased support and telemonitoring arms. Though telemonitoring increased hospitalization for $\mathrm{HF}$, it decreased length of stay, probably due to a higher comfort level of healthcare providers being able to monitor patients at home. Finally, the Specialized Primary and Networked Care in Heart Failure-II (SPAN CHF-II) trial evaluated the impact of automated home monitoring added to a disease management program and found no added benefit for providing daily medication prompts, recordings of weight, vital signs, and symptoms. ${ }^{12}$

Though the published meta-analyses show a positive outcome for telemonitoring, they do not include the recent experience from the above-mentioned Tele-HF, TIM-HF, and SPAN-CHF II trials. At this time, the strength of evidence in favor of these interventions is unclear. Moreover, it is difficult to interpret the meta-analyses of studies that had varied interventions clubbed under telemonitoring.

\section{Monitoring With Specially Designed Wearable and Implantable Technology}

The focus of telemonitoring has centered on predicting acute decompensation episodes that are typically associated with fluid congestion and require optimization of diuretic therapy. The sine quo non for this process is to identify the parameter that best predicts fluid congestion. As mentioned previously, the sensitivity and specificity of weight as a reflection of total body water is not accurate. Parameters beyond simple vital signs and weight were explored in the Multi-Sensor Monitoring in Congestive Heart Failure (MUSIC) study, ${ }_{1}^{13}$ which used a multi-sensor noninvasive external monitoring device to measure and transmit bioimpedance, heart rate, respiratory rate and volume, activity duration and intensity, and body posture. The investigators utilized a development cohort to identify a single or a multiparameter algorithm with three components: fluid index, breath index, and personalization parameters. Using all three parameters yielded a sensitivity of $65 \%$, a specificity of $90 \%$, and a false positive rate of 0.7 events per patient-year. Though this technology seems quiet promising as a continuous noninvasive surveillance, the failure rate of the device was approximately $45 \%$, reflecting the need for further enhancements. Additionally, the concept of a wearable external device on a constant basis will have compliance issues for widespread use. If feasible, such technology might have a role for a defined period post discharge.

Specialized implantable devices also have been studied with the sole objective of monitoring impedance and arrhythmias to decrease hospitalization. In the Chronicle Offers Management to Patients with Advanced Signs and Symptoms of Heart failure (COMPASS-HF) study, ${ }_{1}^{14}$ a fully implantable device - similar to the pulse generator of a pacemaker - was implanted in patients with both reduced and preserved ejection fraction. This system had the ability to continuously monitor and transmit right ventricular hemodynamic parameters. Of all the hemodynamic data collected, the right ventricular pressure at the time of pulmonary valve opening had a strong correlation with actual pulmonary artery pressures. These data were reviewed at least once a week, and intervention occurred accordingly. There was no significant change in the primary endpoint of HF-related events (hospitalizations, emergency room and urgent care visits needing IV diuretics). A retrospective analysis of the COMPASS-HF data did show a $36 \%$ reduction of a first HF-related hospitalization.
When estimating intracardiac pressures as a surrogate marker of an acute decompensation of HF, it has been shown that the left atrial pressure is an accurate correlate of increased symptoms of shortness of breath. Though the use of continuous hemodynamic monitoring in the inpatient setting has been a controversial topic, it generally is agreed that the estimation of left atrial pressures will lead to early detection of fluid overload state. The recent CHAMPION trial ${ }^{15}$ utilized a wireless sensor deployed into the distal pulmonary artery that could continuously transmit wedge pressure. At 6 months, there was a significant $28 \%$ reduction of the rate of HF-related hospitalizations. The outcome was similar in patients with preserved or low ejection fraction. The safety profile was favorable with a $98.6 \%$ freedom from device-related or system-related complications. Though there was a significant positive impact found in this study, ${ }^{15}$ the device was not approved since the FDA concluded that the positive results of the trial were undermined by the fact that the sponsor intervened to a degree that exceeded the research protocol. ${ }^{16}$

Direct left atrial pressure monitoring with an implantable wireless technology has been validated in humans by Ritzwema et al. ${ }^{17}$ and studied as a strategy to provide physician guidance of patient self management in the HOMEOSTASIS trial. ${ }^{18}$ An ongoing trial called LAPTOP $^{19}$ is studying the same technology in NYHAIII patients (low and preserved ejection fraction) who are on optimal medical therapy; the objective is to assess if a physiciandirected patient self-management system guided by left atrial pressure will reduce HF hospitalizations. The study is expected to be complete by August 2013.

\section{ICD/CRT Device Diagnostics Monitoring for Heart Failure}

Implantable cardioverter defibrillators (ICD)/cardiac resynchronization therapy (CRT) is mainstay treatment for heart failure patients. Data from 234 hospitals across United States participating in the Get With the Guidelines initiative showed that approximately $25 \%$ of patients hospitalized for heart failure either had or were eligible for an ICD. ${ }^{20}$ Thoracic impedance can be measured with these implanted devices and has been considered as a surrogate marker of pulmonary congestion. When a current is passed between the intracardiac lead and the generator, the lower the impedance to the conduction of this current, the higher the fluid content of the lungs (as fluid is a better conductor than air). In a validation study, Yu et al. ${ }^{21}$ found that the impedance started to decrease 15 days prior to worsening heart failure symptoms. Also, the change in impedance had a high correlation to change in pulmonary capillary wedge pressure in a subset of patients with Swan-Ganz monitoring. Utilizing a threshold value of 60 $\Omega$ days, the authors found $76 \%$ sensitivity and 1.5 false-positive HF decompensations per patient-year of monitoring. In a more recent study of HF patients, Abraham et al. showed that the sensitivity of intrathoracic impedance monitoring was far superior to daily weight monitoring for predicting worsening HF events. ${ }^{22}$ On the contrary, the SENSE-HF trial failed to show utility of the same technology, ${ }^{23}$ and also revealed many practical uncertainties such as unreliability of impedance testing early after implant. The sensitivity to predict HF events was at best $42 \%$ with a positive predictive valve of $38 \%$.

The DOT-HF trial ${ }^{24}$ studied the clinical utility of the OptiVol ${ }^{\circledR}$ (Medtronic, Inc., Minneapolis, MN) implantable monitoring tool to track changes in intrathoracic impedance. The trial was halted early due to under-recruitment. All-cause mortality and rehospitalizations did not change from control to impedancemonitored therapy. Also, post-hoc analysis showed that even if recruitment was optimal, there would not have been a significant 
result. Current efforts are ongoing in the Optilink-HF trial, ${ }^{25}$ where an OptiVol/CareLink ${ }^{\circledR}$ system (Medtronic, Inc., Minneapolis, $\mathrm{MN}$ ) is being used to provide physicians with wireless alerts of threshold deviations for worsening cardiac status. To increase the predictive ability of a diagnostic algorithm, Whellan et al. in the PARTNERS-HF trial ${ }^{26}$ combined more device variables. After censoring device data for the first 60 days, a total of 694 patients were studied from more than 100 centers. Utilizing data from an independent dataset, the authors identified the following criteria: a fluid index $>100 \Omega$ days or any two of the following: (1) long atrial fibrillation duration; (2) rapid ventricular rate during atrial fibrillation; (3) a high ( 260 ) fluid index; (4) low patient activity; (5) high night heart rate; (6) low heart rate variability; (7) low CRT pacing; or (8) ICD shocks. Patients with positive device diagnostics had a hazard ratio of 5.5 (95\% CI 3.4-8.8; $P<0.0001)$ of HF hospitalization with pulmonary congestion within a month. Interestingly, this combined HF device diagnostic algorithm did not provide any kind of risk stratification in those who already had a previous event, making this study less relevant for predicting readmissions.

More recently, Landolina et al. ${ }^{27}$ in the EVOLVO study randomized 200 patients with a Medtronic wireless ICD/CRT-D along with the CareLink network; half had "remote transmission on" while the rest had "remote transmission off." In the control group, audible alerts were turned on with no transmission of data, while in the intervention group, all alerts were transmitted with no audible alerts. The majority of patients had NYHA II heart failure symptoms. The transmitted data were reviewed at least once daily. The primary endpoint was emergency department or urgent in-office visits, which were then broken down into a secondary endpoint of visits related to episodes of worsening HF and those for arrhythmias or ICD-related events. Patients in the remote-monitoring arm had significantly less events (0.59 vs.0.93 events per year; incident rate ratio $0.65 ; 95 \%$ CI $0.49-0.88 ; P=0.005$ ). In fact, the primary endpoint was driven by the difference in visits for episodes of worsening HF. As expected with a directed intervention, the median time from the alert condition to ICD data review was 24.8 days for the standard care arm compared to 1.4 days in the remote monitoring arm. The standard arm patients had four in-office visits per year while those in the remote monitoring arm had only two planned in-office visits. Another key point of this study is that the system of providing audible alerts from the ICD/CRT-D devices did not make much of a difference, which suggests that relying on the patient to react appropriately is not a feasible option. In fact, prior studies have shown that many alerts go unnoticed by patients. ${ }^{28,} 29$

These studies reflect the important role of device diagnostics in the ever-growing population of HF patients with an implanted ICD or CRT device. Future studies should take into account the limitations of this technology and the variations in the specificity of impedance information.

\section{Home Monitoring - Is it an Epidemiologically Feasible Solution?}

A significant challenge posed by continuous monitoring of HF patients is the burden of information overload. Resources to manage and act on the transmitted information from these patients are vital to the success of home monitoring. Studies have shown that the amount of information in a controlled, limited-time trial setting already seems overwhelming. The legal implications of timely follow-up of continuously monitored information and the scope of false positives with health care utilizations is a daunting aspect for handling the information. Moreover, the cost of phone monitoring with no reimbursements might make this modality less lucrative as opposed to using already available ICD/CRT-D technology. Also, the presence of multiple vendors and proprietary algorithms of each device company poses a challenge in creating a universally simplified approach to implement a structured algorithm. For those who do not need an implantable device, advancements in wearable monitors and Bluetooth transmission of information seems promising, yet with no strong evidence. Patient compliance issues with these technologies might be overcome by emerging piezo crystal sensors. Recently, Benett et al. ${ }^{30}$ reported the feasibility of using the EarlySense's EverOn ${ }^{\circledR}$ (EarlySense, Waltham, MA) under-the-mattress sensor system to track physiological information such as respiratory rate, heart rate, and movement rate during sleep in three patients. Also, advancements in implantable wireless technology seen with the pulmonary capillary pressure monitoring device CardioMEMS ${ }^{\circledR}$ (CardioMEMS, Inc., Atlanta, GA) and the left atrial pressure monitor HeartPOD ${ }^{\mathrm{TM}}$ System (St. Jude Medical, Inc., St. Paul, MN) or Promote ${ }^{\circledR}$ LAP System (St. Jude Medical, Inc., St. Paul, MN) bring us closer to finding the holy grail of home monitoring systems.

Attempts at shifting the burden of self management to patients have not been very effective due to the complexity of the therapies and the advancing age of HF patients in the United States. Between $27-44 \%$ of Medicare enrollees have marginal or inadequate health literacy, ${ }^{31}$ making this task more challenging. Powell et al. ${ }^{32}$ in the HART study found that the addition of self-management counseling to an educational intervention did not make a difference in death or HF hospitalization in patients with mild to moderate HF. Earlier smaller studies also have not shown any convincing evidence for self-management. ${ }^{33-36}$ Therefore, a strategy that minimizes patient responsibility in monitoring and care might be more pragmatic. All strategies should still aim at having a patient-centered care plan along with stressing patient education. ${ }^{37}$ Moreover, there is overwhelming evidence that a multidisciplinary approach to HF care reduces hospital readmissions and improves outcomes in these patients. ${ }^{38}$ Hence, it is recommended in both U.S. and European guidelines. ${ }^{39,40}$ Irrespective of the parameters and modes of home monitoring, a patient-tailored and disease management approach needs to be a complimentary aspect of any initiative to minimize healthcare burden and readmissions. More importantly, it stresses the fact that perhaps we should not wait for a readmission but, rather, focus on the first admission by developing outpatient strategies that include quality assurance in the outpatient clinic setting and effective education for the patient, caregiver, and community at large. The focus of all remote monitoring seems to be skewed towards avoiding acute exaggerations and optimizing diuretics. A more sustainable impact could be made by focusing on effective uptitration of medical therapy upon discharge or new diagnosis. The explosion of social media and smart phone applications is a potentially untapped resource in creating a patient centered system.

\section{Conclusion}

The need to create innovative care systems for HF patients is obvious from the increasing health care burden of this rampant disease. Cost-effectiveness analyses for the most part have been favorable towards remote monitoring. ${ }^{41,42}$ The effectiveness and cost investments will vary based on the technology adapted and the system in place for handling the monitored patient information. The current evidence is not overwhelming for certain basic technologies, and evidence for the others is emerging. While remote monitoring has not yet reached its prime time, the advancements show promise for the future. 
Conflict of Interest Disclosure: The author has completed and submitted the Methodist DeBakey Cardiovascular Journal Conflict of Interest Statement and none were reported.

Funding/Support: The author has no funding disclosures.

Keywords: heart failure, home monitoring, telemonitoring, remote monitoring, disease management

\section{References}

1. Krumholz HM, Parent EM, Tu N, Vaccarino V, Wang Y, Radford $\mathrm{MJ}$, et al. Readmission after hospitalization for congestive heart failure among Medicare beneficiaries. Arch Intern Med. 1997 Jan 13;157(1):99-104.

2. Ferrante D, Varini S, Macchia A, Soifer S, Badra R, Nul D, et al.; GESICA Investigators. Long-term results after a telephone intervention in chronic heart failure: DIAL (Randomized Trial of Phone Intervention in Chronic Heart Failure) follow-up. J Am Coll Cardiol. 2010 Jul 27;56(5):372-8.

3. Grancelli $H$, Varini S, Ferrante D, Schwartzman R, Zambrano C, Soifer S, et al.; GESICA Investigators. Randomized Trial of Telephone Intervention in Chronic Heart Failure (DIAL): study design and preliminary observations. J Card Fail. 2003 Jun;9(3):172-9.

4. GESICA Investigators. Randomised trial of telephone intervention in chronic heart failure: DIAL trial. BMJ. 2005 Aug 20;331(7514):425.

5. Inglis SC, Clark RA, McAlister FA, Stewart S, Cleland JG. Which components of heart failure programmes are effective? A systematic review and meta-analysis of the outcomes of structured telephone support or telemonitoring as the primary component of chronic heart failure management in 8323 patients: Abridged Cochrane Review. Eur J Heart Fail. 2011 Sep;13(9):1028-40.

6. Jaarsma, T, van der Wal MH, Lesman-Leegte I, Luttik ML, Hogenhuis J, Veeger $\mathrm{NJ}$, et al. Effect of moderate or intensive disease management program on outcome in patients with heart failure: Coordinating Study Evaluating Outcomes of Advising and Counseling in Heart Failure (COACH). Arch Intern Med. 2008 Feb 11;168(3):316-24.

7. Cleland JG, Louis AA, Rigby AS, Janssens U, Balk AH; TEN-HMS Investigators. Noninvasive home telemonitoring for patients with heart failure at high risk of recurrent admission and death: the Trans-European Network-Home-Care Management System (TEN-HMS) study. J Am Coll Cardiol. 2005 May 17;45(10):1654-64.

8. Inglis SC, Clark RA, McAlister FA, Ball J, Lewinter C, Cullington $D$, et al. Structured telephone support or telemonitoring programmes for patients with chronic heart failure. Cochrane Database Syst Rev. 2010 Aug 4;(8):CD007228.

9. Zhang J, Goode KM, Cuddihy PE, Cleland JG; TEN-HMS Investigators. Predicting hospitalization due to worsening heart failure using daily weight measurement: analysis of the Trans-European Network-Home-Care Management System (TEN-HMS) study. Eur J Heart Fail. 2009 Apr;11(4):420-7.

10. Goldberg LR, Piette JD, Walsh MN, Frank TA, Jaski BE, Smith AL, et al.; WHARF Investigators. Randomized trial of a daily electronic home monitoring system in patients with advanced heart failure: the Weight Monitoring in Heart Failure (WHARF) trial. Am Heart J. 2003 Oct;146(4):705-12.

11. Heart Failure Society of America [Internet]. St. Paul, MN: Heart Failure Society of America; 2012. Self-care: following your treatment plan and dealing with your symptoms; 2003 [cited 2012 Dec 18]. Available from: http://www.hfsa.org/ pdf/module4.pdf.
12. Konstam V, Gregory D, Chen J, Weintraub A, Patel A, Levine D, et al. Health-related quality of life in a multicenter randomized controlled comparison of telephonic disease management and automated home monitoring in patients recently hospitalized with heart failure: SPAN-CHF II trial. J Card Fail. 2011 Feb;17(2):151-7.

13. Anand IS, Tang WH, Greenberg BH, Chakravarthy N, Libbus I, Katra RP; Music Investigators. Design and performance of a multisensor heart failure monitoring algorithm: results from the multisensor monitoring in congestive heart failure (MUSIC) study. J Card Fail. 2012 Apr;18(4):289-95.

14. Bourge RC, Abraham WT, Adamson PB, Aaron MF, Aranda JM Jr, Magalski A, et al. Randomized controlled trial of an implantable continuous hemodynamic monitor in patients with advanced heart failure: the COMPASS-HF study. J Am Coll Cardiol. 2008 Mar 18;51(11):1073-9.

15. Abraham WT, Adamson PB, Bourge RC, Aaron MF, Costanzo MR, Stevenson LW, et al.; CHAMPION Trial Study Group. Wireless pulmonary artery haemodynamic monitoring in chronic heart failure: a randomised controlled trial. Lancet. 2011 Feb 19;377(9766):658-66.

16. U.S. Food and Drug Administration (Internet]. Silver Spring, MD: U.S. Department of Health and Human Services; c2012. Inspections, Compliance, Enforcement, and Criminal Investigations; CardioMEMS, Inc. Warning letter; 2012 Jun 5 [cited 2013 Jan 16]. Available from: http://www.fda.gov/ICECl/ EnforcementActions/WarningLetters/2012/ucm320624.htm.

17. Ritzema J, Melton IC, Richards AM, Crozier IG, Frampton C, Doughty RN, et al. Direct left atrial pressure monitoring in ambulatory heart failure patients: initial experience with a new permanent implantable device. Circulation. 2007 Dec 18;116(25):2952-9.

18. Ritzema J, Troughton R, Melton I, Crozier I, Doughty R, Krum $\mathrm{H}$, et al. Physician-directed patient self-management of left atrial pressure in advanced chronic heart failure. Circulation. 2010 Mar 9;121(9):1086-95.

19. ClinicalTrials.gov [Internet]. Bethesda, MD: National Institutes of Health; c2012. Left Atrial Pressure Monitoring to Optimize Heart Failure Therapy (LAPTOP-HF); 2012 [cited 2012 Dec 28]. Available from: http://clinicaltrials.gov/ct2/show/ NCT01121107?term=LAPTOP-HF\&rank=1.

20. Shah B, Hernandez AF, Liang L, Al-Khatib SM, Yancy CW, Fonarow, GC, et al.; Get With The Guidelines Steering Committee. Hospital variation and characteristics of implantable cardioverter-defibrillator use in patients with heart failure: data from the GWTG-HF (Get With The Guidelines-Heart Failure) registry. J Am Coll Cardiol. 2009 Feb 3;53(5):416-22.

21. Yu CM, Wang L, Chau E, Chan RH, Kong SL, Tang MO, et al. Intrathoracic impedance monitoring in patients with heart failure: correlation with fluid status and feasibility of early warning preceding hospitalization. Circulation. 2005 Aug 9;112(6):841-8.

22. Abraham WT, Compton S, Haas G, Foreman B, Canby $\mathrm{RC}$, Rishel R, et al. Intrathoracic impedance vs daily weight monitoring for predicting worsening heart failure events: results of the Fluid Accumulation Status Trial (FAST). Congest Heart Fail. 2011 Mar-Apr;17(2):51-5.

23. Conraads VM, Tavazzi L, Santini M, Oliva F, Gerritse B, Yu CM, et al. Sensitivity and positive predictive value of implantable intrathoracic impedance monitoring as a predictor of heart failure hospitalizations: the SENSE-HF trial. Eur Heart J. 2011 Sep;32(18):2266-73. 
24. van Veldhuisen DJ, Braunschweig F, Conraads V, Ford I, Cowie MR, Jondeau G, et al.; DOT-HF Investigators. Intrathoracic impedance monitoring, audible patient alerts, and outcome in patients with heart failure. Circulation. 2011 Oct 18;124(16):1719-26.

25. Brachmann J, Böhm M, Rybak K, Klein G, Butter C, Klemm $\mathrm{H}$, et al. Fluid status monitoring with a wireless network to reduce cardiovascular-related hospitalizations and mortality in heart failure: rationale and design of the OptiLink HF Study (Optimization of Heart Failure Management using OptiVol Fluid Status Monitoring and CareLink). Eur J Heart Fail. 2011 Jul;13(7):796-804.

26. Whellan DJ, Ousdigian KT, Al-Khatib SM, Pu W, Sarkar S, Porter CB, et al.; PARTNERS Study Investigators. Combined heart failure device diagnostics identify patients at higher risk of subsequent heart failure hospitalizations: results from PARTNERS HF (Program to Access and Review Trending Information and Evaluate Correlation to Symptoms in Patients With Heart Failure) study. J Am Coll Cardiol. 2010 Apr 27;55(17):1803-10.

27. Landolina M, Perego GB, Lunati M, Curnis A, Guenzati G, Vicentini $A$, et al. Remote monitoring reduces healthcare use and improves quality of care in heart failure patients with implantable defibrillators: the evolution of management strategies of heart failure patients with implantable defibrillators (EVOLVO) study. Circulation. 2012 Jun 19;125(24):2985-92.

28. Simons EC, Feigenblum DY, Nemirovsky D, Simons GR. Alert tones are frequently inaudible among patients with implantable cardioverter-defibrillators. Pacing Clin Electrophysiol. 2009 Oct;32(10):1272-5.

29. Knackstedt C, Mischke K, Schimpf T, Waringer J, Fache K, Frechen D, et al. Integration of automatic intrathoracic fluid content measurement into clinical decision making in patients with congestive heart failure. Pacing Clin Electrophysiol. 2008 Aug;31(8):961-7.

30. Bennett MK, Gorodeski EZ. Home monitoring using a novel under-the-mattress Piezoelectric sensor. Poster session presented at: 16th Annual Scientific Meeting of the Heart Failure Society of America; 2012 Sep 9-12; Seattle, WA.

31. Gazmararian JA, Baker DW, Williams MV, Parker RM, Scott TL, Green DC, et al. Health literacy among Medicare enrollees in a managed care organization. JAMA. 1999 Feb10;281(6):545-51.

32. Powell LH, Calvin JE Jr, Richardson D, Janssen I, Mendes de Leon CF, Flynn KJ, et al.; HART Investigators. Self-management counseling in patients with heart failure: the heart failure adherence and retention randomized behavioral trial. JAMA. 2010 Sep 22;304(12):1331-8.

33. Harrison MB, Browne GB, Roberts J, Tugwell P, Gafni A, Graham ID. Quality of life of individuals with heart failure: a randomized trial of the effectiveness of two models of hospitalto-home transition. Med Care. 2002 Apr;40(4):271-82.

34. Wright SP, Walsh $H$, Ingley KM, Muncaster SA, Gamble GD, Pearl A, et al. Uptake of self-management strategies in a heart failure management programme. Eur J Heart Fail. 2003 Jun;5(3):371-80.
35. Prasun MA, Kocheril AG, Klass PH, Dunlap SH, Piano MR. The effects of a sliding scale diuretic titration protocol in patients with heart failure. J Cardiovasc Nurs. 2005 Jan-Feb;20(1):62-70.

36. DeWalt DA, Malone RM, Bryant ME, Kosnar MC, Corr KE, Rothman $\mathrm{RL}$, et al. A heart failure self-management program for patients of all literacy levels: a randomized, controlled trial [ISRCTN11535170]. BMC Health Serv Res. 2006 Mar13;6:30.

37. Krumholz HM, Amatruda J, Smith GL, Mattera JA, Roumanis SA, Radford MJ, et al. Randomized trial of an education and support intervention to prevent readmission of patients with heart failure. J Am Coll Cardiol. 2002 Jan 2;39(1):83-9.

38. McAlister FA, Stewart S, Ferrua S, McMurray JJ. Multidisciplinary strategies for the management of heart failure patients at high risk for admission: a systematic review of randomized trials. J Am Coll Cardiol. 2004 Aug 18;44(4):810-9.

39. Hunt SA, Abraham WT, Chin MH, Feldman AM, Francis GS, Ganiats TG, et al.; American College of Cardiology; American Heart Association Task Force on Practice Guidelines; American College of Chest Physicians; International Society for Heart and Lung Transplantation; Heart Rhythm Society. ACC/AHA 2005 Guideline Update for the Diagnosis and Management of Chronic Heart Failure in the Adult: a report of the American College of Cardiology/American Heart Association Task Force on Practice Guidelines (Writing Committee to Update the 2001 Guidelines for the Evaluation and Management of Heart Failure): developed in collaboration with the American College of Chest Physicians and the International Society for Heart and Lung Transplantation: endorsed by the Heart Rhythm Society. Circulation. 2005 Sep 20;112(12):e154-235.

40. Dickstein K, Cohen-Solal A, Filippatos G, McMurray JJ, Ponikowski P, Poole-Wilson PA, et al.; ESC Committee for Practice Guidelines (CPG). ESC guidelines for the diagnosis and treatment of acute and chronic heart failure 2008: the Task Force for the diagnosis and treatment of acute and chronic heart failure 2008 of the European Society of Cardiology. Developed in collaboration with the Heart Failure Association of the ESC (HFA) and endorsed by the European Society of Intensive Care Medicine (ESICM). Eur J Heart Fail. 2008 Oct;10(10):933-89.

41. Klersy C, De Silvestri A, Gabutti G, Raisaro A, Curti M, Regoli $F$, et al. Economic impact of remote patient monitoring: an integrated economic model derived from a meta-analysis of randomized controlled trials in heart failure. Eur $\mathrm{J}$ Heart Fail. 2011 Apr;13(4):450-9.

42. Pekmezaris R, Mitzner I, Pecinka KR, Nouryan CN, Lesser $\mathrm{ML}$, Siegel M, et al. The impact of remote patient monitoring (telehealth) upon Medicare beneficiaries with heart failure. Telemed J E Health. 2012 Mar;18(2):101-8. 